01

\title{
Моделирование демпферов из вязкоупругих материалов
}

\author{
() Ю.В. Максимов, Ю.С. Легович, Д.Ю. Максимов \\ Институт проблем управления им. В.А. Трапезникова РАН, \\ 117997 Москва, Россия \\ e-mail: dmmax@inbox.ru
}

Поступило в Редакцию 30 июля 2020 г.

В окончательной редакции 10 сентября 2020 г.

Принято к публикации 11 сентября 2020 г.

\begin{abstract}
Учет демпфирования в задачах динамики конструкций - важная и нетривиальная проблема. Ее сложность не в последнюю очередь обусловлена необходимостью задания верных данных используемых материалов и выбора подходящей для анализа модели. В работе рассмотрены некоторые модели вязкоупругих материалов с точки зрения возможностей применения этих моделей для гармонического анализа демпфирующих свойств различных материалов в линейной области упругих деформаций. Предлагаемый анализ основан на использовании параметров вязкоупругих материалов, заданных в виде коэффициентов дифференциального уравнения малых вынужденных колебаний. Показано, что для рассмотренных моделей характерна различная частотная зависимость параметров моделируемых материалов. Это открывает возможность скомбинировать модель с частотными характеристиками ее параметров, приближающимися к частотным характеристикам параметров исследуемых вязкоупругих материалов.
\end{abstract}

Ключевые слова: вибрации, демпфирование в квадрокоптерах, модели и параметры вязкоупругих материалов, частотные характеристики вязкоупругих материалов.

DOI: 10.21883/JTF.2021.03.50514.239-20

\section{Введение}

Источниками вибраций в динамических (механических) конструкциях очень часто являются вращающиеся элементы, дисбаланс или изменение режима работы которых и вызывают нежелательные вибрации. Нежелательные как для самой конструкции, так и для окружающей среды. Крайне негативными могут быть последствия резонансных явлений, проявляющихся, когда частота возбуждаемых колебаний приближается к резонансной частоте механической системы. Чем и объясняется важность исследования частотных свойств проектируемых конструкций еще на этапе моделирования. Особенно для конструкций, в которых частоты источников вибраций изменяются в широком диапазоне. Ярким примером таких систем являются квадрокоптеры (мультикоптеры), в которых источниками вибраций являются двигатели с тянущими винтами, частота вращения которых может изменяться в широких пределах, а элемент, чувствительный к вибрациям (управляющий контроллер), располагается на той же раме, что и двигатели. Амортизаторы навесного оборудования в квадрокоптерах зачастую выполняются из вязкоупругих ${ }^{1}$ материалов. Одним из часто используемых материалов является резина, которая благодаря своим свойствам находит широкое применение для демпфирования колебаний в различных механических конструкциях [2], в том числе и в авиастроении. Так, например, резина является основным демпфирующим

\footnotetext{
${ }^{1}$ В вязкоупругих материалах напряжения и деформации характеризуются рассеянием энергии на замкнутом цикле деформации и постепенным исчезновением деформации при полном снятии нагрузок [1].
}

элементом в многослойной структуре такого важного узла легкого вертолета нового поколения, как торсион, соединяющего лопасть несущего винта с валом [3].

Необходимость демпфирования нежелательных вибраций остро ставит вопрос о корректном моделировании демпфирующих устройств из вязкоупругих материалов. Моделирование вязкоупругих материалов основано на линейной теории вязкоупругости материалов с памятью. Линейная теория вязкоупругости сформировалась на базе представлений о таких свойствах твердых тел, как пластичность и ползучесть [4-7]. Интенсивность исследований свойств вязкоупругих материалов, в том числе специально разрабатываемых для демпфирования колебаний в различных механических конструкциях, увеличивается. Появляются и новые монографии [8], и многочисленные работы по отдельным проблемам как теоретического, так и практического характера [3,9-13]. В литературе описаны подходы к оценке демпфирующих свойств материалов, используемых для этих целей, и различные их модели $[4,5,8,14]$. Однако критериев оценки степени соответствия моделей конкретным материалам не выработано. Зачастую отсутствуют и данные о параметрах демпфирующих материалов, необходимые для их моделирования. При этом особую важность приобретает вопрос о корректном измерении параметров вязкоупругих материалов [12,13].

В настоящей работе на примерах некоторых известных моделей вязкоупругих материалов показаны различия их частотных свойств и возможность использования частотной зависимости параметров демпфирующих ма- 
териалов в качестве критерия при подборе параметров подходящей модели.

\section{1. Вынужденные колебания механической системы. Уравнение и модель Фойгта}

Дифференциальное уравнение малых вынужденных колебаний имеет вид

$$
x^{\prime \prime}+r x^{\prime}+k x=F(t),
$$

где $F(t)$ - обобщенная периодическая внешняя сила, сопряженная с обобщенной координатой $x ; x^{\prime}, x^{\prime \prime}-$ первая и вторая производные $x$ по $t$ соответственно; $m, r$ и $k$ - обобщенные коэффициенты инерции, трения и упругости соответственно.

Внешней силе, таким образом, противодействуют три силы:

сила инерции:

$$
F_{m}(t)=m x^{\prime \prime},
$$

где $m$ - масса тела, к которому приложена сила, $F_{r}(t)-$ обобщенная сила трения:

$$
F_{r}(t)=r x^{\prime},
$$

где $r$ - обобщенный коэффициент трения и $F_{k}(t)-$ квазиупругая сила:

$$
F_{k}(t)=k x
$$

где $k$ - коэффициент квазиупругой силы.

Уравнение (1) представляют также в виде

$$
x^{\prime \prime}+2 \delta x^{\prime}+\Omega_{0}^{2} x=\frac{1}{m} F(t),
$$

где $\delta$ и $\Omega_{0}-$ коэффициент затухания и частота свободных колебаний системы соответственно:

$$
\begin{aligned}
\delta & =\frac{r}{2 m}, \\
\Omega_{0} & =\sqrt{\frac{k}{m}} .
\end{aligned}
$$

При моделировании механических систем широко используется представление ее динамической модели в виде системы с сосредоточенными параметрами $[15,16]$, которая во многих случаях с достаточной степенью точности отражает наиболее существенные факторы, определяющие поведение системы при заданных воздействиях. При построении таких моделей исходят из того, что инерционные свойства механизма предполагаются сосредоточенными в отдельных точках в виде приведенных масс, и эти точки связаны между собой упругими, диссипативными и геометрическими безынерционными связями [16].

При таком подходе изображение механической системы, вынужденные колебания которой описываются

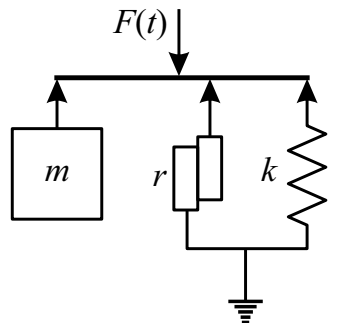

Рис. 1. Схематическое изображение системы, совершающей вынужденные колебания.

уравнением (1), схематически может быть представлено в виде, показанном на рис. 1. На нем использованы следующие обозначения: $F(t)$ - сила, действующая на 3 элемента: $m$ - инерционный элемент массой $m ; r$ диссипативный элемент с коэффициентом трения $r ; k-$ упругий элемент с коэффициентом упругости $k$.

В таком изображении вязкоупругий материал (демпфер) представляется в виде модели Фойгта [5]. В рассматриваемом случае (гармонического анализа) возмущающая сила изменяется по гармоническому закону:

$$
F(t)=F_{0}+F_{a} \cos (\Omega t),
$$

где $F_{0}$ и $F_{a}$ - постоянная составляющая и амплитуда гармонической составляющей внешней силы соответственно; $\Omega$ - круговая частота гармонической составляющей действующей силы.

Установившиеся вынужденные колебания также являются гармоническими (такой же частоты, но сдвинутые по фазе относительно внешней силы):

$$
x=X_{0}+X_{a} \cos (\Omega t+\varphi),
$$

где $X_{0}$ и $X_{a}$ - постоянная составляющая, и амплитуда гармонической составляющей смещения $x$ под действием силы $F(t)$ соответственно; $\varphi$ - фазовый сдвиг смещения относительно действующей силы; $X_{0}=\frac{F_{0}}{k}$.

Амплитуда колебаний $X_{a}$ и фазовый сдвиг $\varphi$ зависят от частоты колебаний:

$$
\begin{gathered}
X_{a}(\Omega)=\frac{F_{a}}{m \sqrt{\left(\Omega_{0}^{2}-\Omega^{2}\right)^{2}+(2 \delta \Omega)^{2}}}, \\
\tan \varphi(\Omega)=-\frac{2 \delta \Omega}{\Omega_{0}^{2}-\Omega^{2}} .
\end{gathered}
$$

В данном случае (гармоническое воздействие)

$$
\begin{aligned}
x^{\prime}= & -X_{a}(\Omega) \Omega \sin (\Omega t+\varphi(\Omega))= \\
& -X_{a}(\Omega) \Omega \cos (\Omega t-\beta(\Omega)),
\end{aligned}
$$

где $\beta(\Omega)$ - сдвиг фаз между силой $F(t)$ и скоростью изменения смещения $x$

$$
\begin{gathered}
\beta(\Omega)=\frac{\pi}{2}-\varphi(\Omega) \\
\tan \beta(\Omega)=-\tan ^{-1} \varphi(\Omega)=\frac{\Omega_{0}^{2}-\Omega^{2}}{2 \delta \Omega}
\end{gathered}
$$




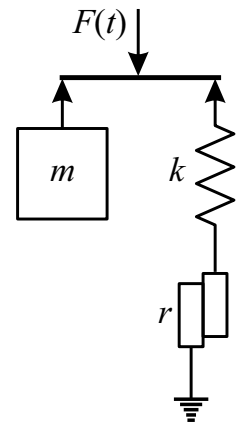

Рис. 2. Модель Максвелла в системе, совершающей вынужденные колебания.

$$
x^{\prime \prime}=-X_{a}(\Omega) \Omega^{2} \cos (\Omega t+\varphi(\Omega)) .
$$

Диссипативные потери установившихся вынужденных колебаний, характеризуемые мощностью потерь $P_{d}$, описываются диссипативной функцией $\Phi$ [17]:

$$
P_{d}=2 \Phi ; \quad \Phi=\frac{r\left(x^{\prime}\right)^{2}}{2}
$$

Потери энергии за период колебаний $W_{d}(T)$ (где $T=2 \pi / \Omega)$ составляют

$$
W_{d}(T)=\int_{0}^{T} 2 \Phi d t=r \int_{0}^{T}\left(x^{\prime}\right)^{2} d t
$$

Выполнив интегрирование, получим

$$
W_{d}(T)=r \pi \Omega X_{a}^{2}=r \pi \Omega \frac{F_{a}^{2}}{m^{2}\left[\left(\Omega_{0}^{2}-\Omega^{2}\right)^{2}+(2 \delta \Omega)^{2}\right]} .
$$

Частотная зависимость амплитуды колебания и мощности потерь имеет резонанс вблизи $\Omega \approx \Omega_{0}$ при $\delta<\Omega_{0} / \sqrt{2}[4]$.

\section{2. Модели Максвелла и Кельвина-Фойгта}

Модель Максвелла показана на рис. 2. В данном случае внешней силе $F(t)$ противодействуют две силы:

- сила инерции $F_{1}(t)=F_{m}(t)=m x^{\prime \prime}$, где $m$ - масса тела, к которому приложена сила;

- сила демпфирования $F_{2}(t)$, создаваемая последовательно соединенными элементами трения и упругости.

При этом деформации ${ }^{2}$ элементов трения и упругости ( $x_{r}$ и $x_{k}$ соответственно) определяются силой $F_{2}(t)=F_{2} \cos (\Omega t+\psi)$, где символом $\psi$ обозначен фазо-

\footnotetext{
2 Здесь и далее термином ,деформация“ обобщенно обозначены деформация и смещение элементов модели - пружины, подвергающейся растяжению и сжатию, и поршня, смещающегося относительно некоторого корпуса.
}

вый сдвиг силы $F_{2}(t)$ относительно силы $F(t)^{3}$ :

$$
\begin{aligned}
& F_{2}(t)=r x_{r}^{\prime}, \\
& F_{2}(t)=k x_{k},
\end{aligned}
$$

где $x_{r}^{\prime}$ - производная смещения (деформации) элемента трения.

Полная деформация демпфера $x_{2}$ равна полному смещению $x$ : $x_{2}=x_{r}+x_{k}=x$. При этом (с учетом (6) и (7))

$$
\begin{gathered}
x_{r}=\frac{F_{2}}{\Omega r} \sin (\Omega t+\psi), \\
x_{k}=\frac{F_{2}}{k} \cos (\Omega t+\psi), \\
x_{2}=F_{2}\left[\frac{1}{\Omega r} \sin (\Omega t+\psi)+\frac{1}{k} \cos (\Omega t+\psi)\right] .
\end{gathered}
$$

После преобразования (8) получим

$$
x_{2}=F_{2} \sqrt{\left(\frac{1}{\Omega r}\right)^{2}+\left(\frac{1}{k}\right)^{2}} \cos (\Omega t+\psi-\xi),
$$

где $\xi$ определяется из выражения:

$$
\tan \xi=\frac{k}{\Omega r} .
$$

Для сравнения характеристик моделей Максвелла и Фойгта удобно преобразовать модель Максвелла к виду модели Фойгта (рис. 1) с соответствующими (эквивалентными) параметрами. При таком преобразовании сила $F_{2}(t)$ представляется в виде суммы двух ортогональных составляющих $F_{2 r}(t)$ и $F_{2 k}(t)$. Векторная диаграмма, представленная на рис. 3 , отображает соотношение всех взаимодействующих сил.

На рис. 3 амплитуды векторов обозначены так же, как амплитуды соответствующих сил. Символ $\varphi$ обозначает фазовый сдвиг смещения $x$ относительно действующей силы $F(t)$, символ $\psi$ обозначает фазовый сдвиг силы $F_{2}(t)$ относительно силы $F(t)$. Сила инерции $F_{1}(t)=F_{m}(t)$ противофазна смещению. Сила упругости $F_{2 k}(t)$ (как составляющая силы $\left.F_{2}(t)\right)$ синфазна смещению. Сила трения $F_{2 r}(t)$ (другая составляющая силы $\left.F_{2}(t)\right)$ ортогональна смещению (сдвинута по фазе относительно смещения на $90^{\circ}$ ):

$$
\begin{gathered}
F_{2}=\sqrt{F_{2 k}^{2}+F_{2 r}^{2}}, \\
F_{2 k}=F_{a} \cos \varphi+F_{m}, \\
F_{2 r}=F_{a} \sin \varphi .
\end{gathered}
$$

Так как модель Максвелла, трансформированная к виду модели Фойгта, должна все же работать так же, как и до трансформации, т. е. обеспечивать такую же реакцию на то же самое воздействие, то в трансформированной

\footnotetext{
3 Здесь и далее амплитуды сил обозначаются теми же символами, что и силы, но без указания зависимости от времени $t$, только амплитуда силы $F(t)$ обозначена $F_{a}$; то же относительно смещений.
} 


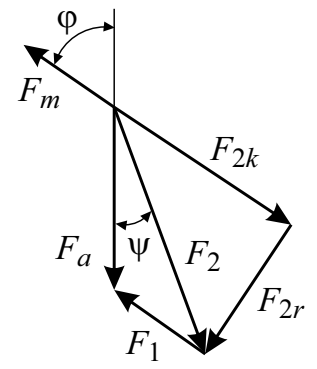

Рис. 3. Векторная диаграмма взаимодействующих сил.

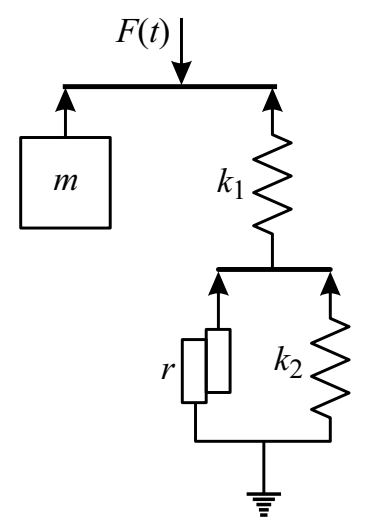

Рис. 4. Модель Кельвина-Фойгта в системе, совершающей вынужденные колебания.

модели параметры ее элементов (коэффициенты трения и упругости) должны измениться определенным образом.

Обозначим коэффициенты трения и упругости в трансформированной модели Максвелла $r_{e}$ и $k_{e}$ соответственно. Тогда с учетом (2), (4) и (5) выражения для $F_{2 k}, F_{2 r}$ и $F_{2}$ могут быть представлены в виде

$$
\begin{gathered}
F_{2 k}(\Omega)=\frac{F_{a} \Omega_{0}^{2}}{\sqrt{\left(\Omega_{0}^{2}-\Omega^{2}\right)^{2}+\left(\frac{\Omega r_{e}}{m}\right)^{2}}}, \\
F_{2 r}(\Omega)=\frac{F_{a} \Omega r_{e}}{m \sqrt{\left(\Omega_{0}^{2}-\Omega^{2}\right)^{2}+\left(\frac{\Omega r_{e}}{m}\right)^{2}}}, \\
F_{2}(\Omega)=F_{a} \sqrt{\frac{\Omega_{0}^{4}+\left(\frac{\Omega r_{e}}{m}\right)^{2}}{\left(\Omega_{0}^{2}-\Omega^{2}\right)^{2}+\left(\frac{\Omega r_{e}}{m}\right)^{2}}} .
\end{gathered}
$$

Выражение для суммарной деформации модели Максвелла (9) с учетом (13) принимает вид

$$
\begin{aligned}
& x_{2}(\Omega)=F_{a} \sqrt{\left(\frac{1}{\Omega r}\right)^{2}+\left(\frac{1}{k}\right)^{2}} \\
& \times \sqrt{\frac{\Omega_{0}^{4}+\left(\frac{\Omega r_{e}}{m}\right)^{2}}{\left(\Omega_{0}^{2}-\Omega^{2}\right)^{2}+\left(\frac{\Omega r_{e}}{m}\right)^{2}}} \cos (\Omega t+\psi-\xi) .
\end{aligned}
$$

При этом амплитуда деформации $X_{2}$ имеет вид

$$
X_{2}(\Omega)=F_{a} \sqrt{\left(\frac{1}{\Omega r}\right)^{2}+\left(\frac{1}{k}\right)^{2}} \sqrt{\frac{\Omega_{0}^{4}+\left(\frac{\Omega r_{e}}{m}\right)^{2}}{\left(\Omega_{0}^{2}-\Omega^{2}\right)^{2}+\left(\frac{\Omega r_{e}}{m}\right)^{2}}} .
$$

Поскольку $x_{2}=x$, то и $X_{2}=X_{a}$, что после преобразования (15) с учетом (3) дает

$$
\Omega_{0}^{4}+\left(\frac{\Omega r_{e}}{m}\right)^{2}=\frac{1}{m^{2}\left[\left(\frac{1}{\Omega r}\right)^{2}+\left(\frac{1}{k}\right)^{2}\right]} .
$$

Для трансформированной модели Максвелла $\Omega_{0}^{2}=k_{e} / m$, что приводит (16) к виду

$$
\left(\frac{\Omega r_{e}}{m}\right)^{2}=\frac{1}{m^{2}\left[\left(\frac{1}{\Omega r}\right)^{2}+\left(\frac{1}{k}\right)^{2}\right]}-\left(\frac{k_{e}}{m}\right)^{2} .
$$

Итак, мы получили одно уравнение, связывающее параметры трансформированной модели Максвелла $r_{e}$ и $k_{e} \mathrm{c}$ параметрами исходной модели $r$ и $k$.

Второе уравнение определяется следующим образом: $F_{2 k}=k_{e} X_{a}$. Выражение (12) определяет составляющую $F_{2 k}$ силы $F_{2}$ как функцию силы $F_{a}$ и параметров $r_{e}$ и $k_{e}$ трансформированной модели Максвелла. Но эта составляющая может быть определена и через параметры $r$ и $k$ исходной модели. А именно

$$
F_{2 k}=F_{2} \cos \xi
$$

что с учетом (13) и (10) дает

$$
F_{2 k}(\Omega)=\frac{F_{a}}{\sqrt{1+\left(\frac{k}{\Omega r}\right)^{2}}} \sqrt{\frac{\Omega_{0}^{4}+\left(\frac{\Omega r_{e}}{m}\right)^{2}}{\left(\Omega_{0}^{2}-\Omega^{2}\right)^{2}+\left(\frac{\Omega r_{e}}{m}\right)^{2}}} .
$$

Приравнивая выражения (12) и (18), после преобразований получим

$$
k_{e}=\frac{\Omega r}{k} \Omega r_{e} .
$$

Уравнения (17) и (19) образуют систему, решение которой относительно $r_{e}$ и $k_{e}$ дает:

$$
\begin{aligned}
& r_{e}=\frac{r}{1+\left(\frac{\Omega r}{k}\right)^{2}}, \\
& k_{e}=\frac{k}{1+\left(\frac{k}{\Omega r}\right)^{2}} .
\end{aligned}
$$

Мы видим, что коэффициенты трансформированной модели в данном случае становятся частотно зависимыми.

Если обратиться к более сложной модели КельвинаФойгта $[8,9]$, показанной на рис. 4 , и трансформировать ее к виду модели Фойгта, то параметры трансформированной модели тоже будут зависеть от частоты характерным для данной модели образом:

$$
r_{e k}=r \frac{\left(\frac{k_{1}}{k_{1}+k_{2}}\right)^{2}}{1+\left(\frac{\Omega r}{k_{1}+k_{2}}\right)^{2}}
$$




$$
k_{e k}=\frac{k_{1} k_{2}}{k_{1}+k_{2}} \frac{1+\frac{(\Omega r)^{2}}{\left(k_{1}+k_{2}\right) k_{2}}}{1+\left(\frac{\Omega r}{k_{1}+k_{2}}\right)^{2}} .
$$

В выражениях (22) и (23) индексами $k_{1}$ и $k_{2}$ обозначены дополнительный упругий элемент и упругий элемент модели Фойгта соответственно (рис. 4).

Частотные зависимости параметров трансформированной модели Кельвина-Фойгта получены с использованием метода электромеханических аналогий [18-20], который существенно упрощает вычисления в сложных случаях. Их вывод приводится в Приложении. Эти зависимости более сложны по сравнению с моделью Максвелла и обладают большей гибкостью с точки зрения возможности их подстройки к частотным зависимостям параметров исследуемого вязкоупругого материала.

\section{3. Пример расчета частотных характеристик}

В качестве примера рассмотрим модели Максвелла и Кельвина-Фойгта резины 57-7024-110, преобразованные к виду модели Фойгта. Результаты измерений релаксационных параметров резины, выполненных при относительной деформации образцов 50\%, представлены в [21]. По этим результатам определены модуль упругости резины на частотах близких к нулю $-E_{0}=1.73 \mathrm{MPa}$ и модуль упругости резины в области высоких частот (стремящихся к бесконечности $)-E_{\infty}=3.6 \mathrm{MPa}$. Параметры преобразованной модели Кельвина-Фойгта на частотах, стремящихся к нулю и к бесконечности, выбраны равными соответствующим параметрам резины на тех же частотах. При выборе параметров модели Максвелла обеспечено равенство коэффициентов упругости модели и материала только в области частот, стремящихся к бесконечности. Для наглядности на рис. 5 и 6 в этом случае представлены примеры частотных зависимостей эквивалентных коэффициентов трения $\left(r_{e}\right.$ и $\left.r_{e k}\right)$ и коэффициентов упругости $\left(k_{e}\right.$ и $\left.k_{e k}\right)$ моделей Максвелла и КельвинаФойгта, преобразованных к виду модели Фойгта.

На рис. 5 и 6 использованы обозначения: $r(M)$ и $k(M)$ - коэффициенты вязкого трения $(r)$ и упругости $(k)$ модели Максвелла соответственно; $r(K F)$, $k 1(K F)$ и $k 2(K F)$ - коэффициенты вязкого трения $(r)$ и упругости ( $k 1$ и $k 2$ ) модели Кельвина-Фойгта соответственно; re и rek - значения эквивалентных коэффициентов вязкого трения $\left(r_{e}\right.$ и $\left.r_{e k}\right)$ моделей Максвелла и Кельвина-Фойгта, преобразованных к виду модели Фойгта, соответственно; ke и kek - значения эквивалентных коэффициентов упругости $\left(k_{e}\right.$ и $\left.k_{e k}\right)$ моделей Максвелла и Кельвина-Фойгта, преобразованных к виду модели Фойгта, соответственно; $f / f n-$ значения нормированных частот.

Нормировка по частоте выполнена таким образом, чтобы среднее значение коэффициента упругости моде-

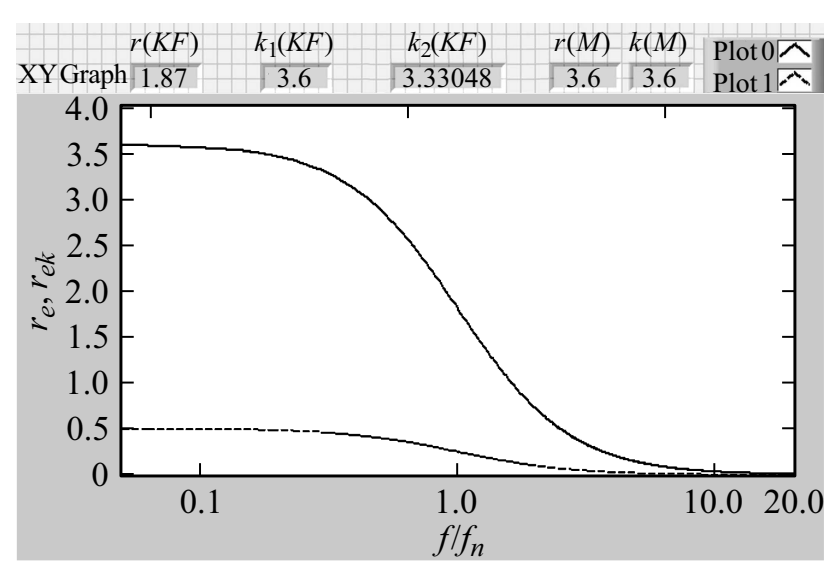

Рис. 5. Пример частотных зависимостей эквивалентных коэффициентов трения моделей Максвелла (сплошная линия) и Кельвина-Фойгта (штриховая линия).

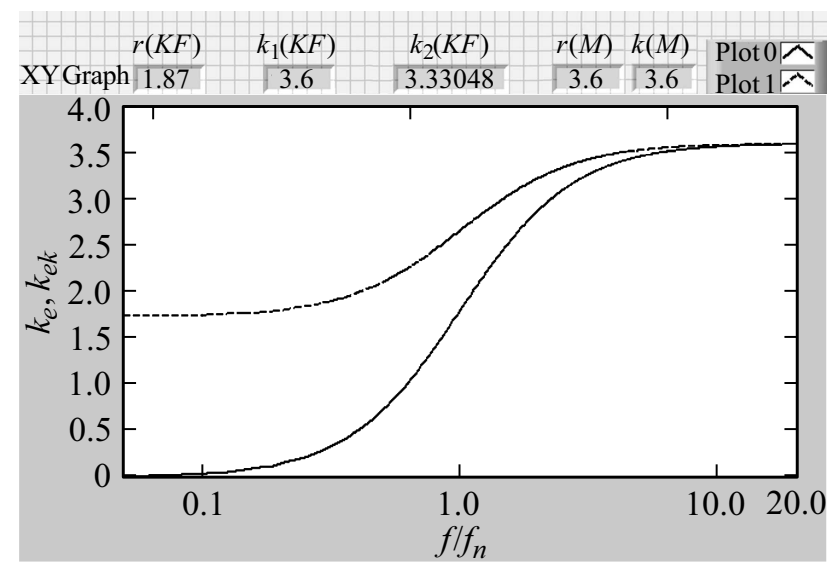

Рис. 6. Пример частотных зависимостей эквивалентных коэффициентов упругости моделей Максвелла (сплошная линия) и Кельвина-Фойгта (штриховая линия).

ли Кельвина-Фойгта для такого материала имело место на частоте $1 \mathrm{~Hz}$.

\section{4. Заключение. Выбор модели вязкоупругого материала}

Рассмотренные модели вязкоупругих материалов, приведенные к одному виду, соответствующему дифференциальному уравнению вынужденных колебаний, характеризуются параметрами, отличающимися своей частотной зависимостью. Так если параметры модели Фойгта частотно независимы, то частотная зависимость параметров моделей Максвелла и Кельвина-Фойгта определяется выражениями (20)-(23).

Выбор подходящей модели для анализа демпфирующих свойств исследуемых материалов непростая задача, поскольку физических механизмов затухания колебаний в реальных материалах множество [14] и, как правило, они неизвестны или не важны в контексте рассматрива- 
емой задачи. Тем не менее независимо от конкретных механизмов затухания моделирование демпфирования колебаний во многих практических задачах является необходимым этапом проектирования механических систем. Проведенный анализ некоторых моделей вязкоупругого тела показывает, что для корректного выбора модели рассматриваемого демпфирующего материала необходимо не только знать (определить) его параметры в некоторых конкретных условиях, но и знать частотную зависимость этих параметров в области частот, характерных для проектируемой системы. Тогда в качестве модели вязкоупругого материала может быть принята модель вида модели Фойгта, но с частотно зависимыми параметрами. Причем частотные зависимости параметров модели и соответствующих параметров реального материала должны быть по возможности близкими друг к другу в заданной области частот. Эта модель может представлять собой трансформацию некоторой модели (какой-либо из известных моделей или впервые синтезированной модели) с элементами, параметры которых не зависят от частоты.

Подводя итоги, отметим следующее.

1. Демпфирующие свойства вязкоупругих материалов в механических конструкциях оцениваются показателями, определяемыми через коэффициенты дифференциального уравнения, описывающего поведение системы при гармоническом воздействии.

2. Эти коэффициенты являются параметрами механической модели Фойгта вязкоупругого материала.

3. В предлагаемой работе показано, что любая механическая модель вязкоупругого материала, состоящая из комбинации частотно независимых элементов вязкого трения и упругости, может быть преобразована к виду модели Фойгта с частотно зависимыми параметрами.

4. В работе предлагается использовать частотные зависимости параметров механических моделей (вязкоупругих материалов), преобразованных к виду модели Фойгта, для оценки степени соответствия модели вязкоупругому материалу путем сравнения частотных зависимостей параметров модели и материала.

5. Для осуществления такого сравнения параметры вязкоупругого материала должны быть выражены через коэффициенты соответствующего дифференциального уравнения.

\section{Приложение}

\section{Применение метода электромеханических аналогий в трансформации модели Кельвина-Фойгта к виду модели Фойгта}

Модель вязкоупругого материала Кельвина-Фойгта и ее электрический аналог показаны на рис. 7, $a, b$ соответственно.

На рис. 7 использованы обозначения: $r_{F}$ и $k_{F}-$ диссипативный и упругий элементы (соответственно)

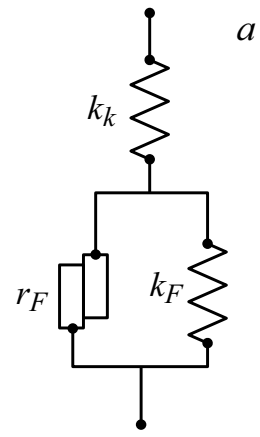

$b$

Рис. 7. Модель Кельвина-Фойгта $(a)$ и ее электрический аналог (b).
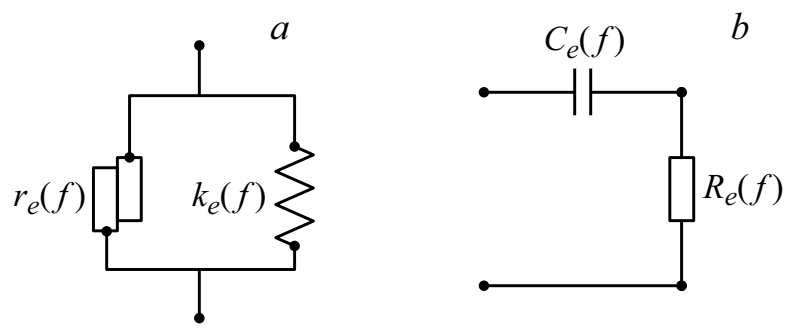

Рис. 8. Модель Фойгта $(a)$ и ее электрический аналог $(b)$ с частотно зависимыми параметрами.

модели Фойгта, входящей в состав модели КельвинаФойгта; $k_{K}$ - дополнительный упругий элемент модели Кельвина-Фойгта; $C_{F}$ и $R_{F}$ - электрическая емкость и сопротивление, являющиеся аналогами элементов $k_{F}$ и $r_{F}$ соответственно; $C_{K}$ - электрическая емкость аналог элемента $k_{K}$.

Bсе элементы модели Кельвина-Фойгта и их электрические аналоги, изображенные на рис. 7, являются частотно независимыми параметрами. Для анализа частотных свойств модели Кельвина-Фойгта удобно воспользоваться ее электрическим аналогом или трансформировать эту модель к виду модели Фойгта с частотно зависимыми параметрами, как показано на рис. 8.

На рисунке использованы обозначения: $C_{e}(f)$ и $R_{e}(f)$ - частотно зависимые элементы (электрическая эмкость и сопротивление соответственно) электрической схемы, эквивалентной схеме, показанной на рис. $7, b ; k_{e}(f)$ и $r_{e}(f)$ - частотно зависимые коэффициенты упругости и трения (соответственно) элементов модели Фойгта, эквивалентной модели КельвинаФойгта, показанной на рис. 7, $a$.

Параметры $k_{e}(f)$ и $r_{e}(f)$ трансформированной модели легко определяются по результатам вычисления параметров $C_{e}(f)$ и $R_{e}(f)$. Для нахождения параметров $C_{e}(f)$ и $R_{e}(f)$ сравним выражения для входного сопротивления двух электрических аналогов, показанных на рис. $7, b$ и $8, b$. 
Выражение для входного сопротивления $Z_{k}$ аналога модели Кельвина-Фойгта имеет вид:

$$
Z_{k}=\frac{1}{Y_{k}}=\frac{1}{j \Omega C_{k}+\frac{1}{R_{F}+j \Omega C_{F}}}
$$

где $Y_{k}$ - входная проводимость электрической схемы - аналога модели Кельвина-Фойгта; $\Omega$ - частота; $C_{k}, R_{F}, C_{F}$ - элементы электрической схемы - аналога модели Кельвина-Фойгта.

Выражение (П1) путем преобразований может быть приведено к виду

$$
\begin{aligned}
Z_{k}= & R_{F} \frac{\left(\frac{C_{F}}{C_{F}+C_{K}}\right)^{2}}{1+\left(\Omega R_{F}\right)^{2}\left(\frac{C_{F} C_{K}}{C_{F}+C_{K}}\right)^{2}} \\
& -j \frac{\frac{1}{\Omega\left(C_{F}+C_{K}\right)}+\Omega C_{K} R_{F}^{2}\left(\frac{C_{F}}{C_{F}+C_{K}}\right)^{2}}{1+\left(\Omega R_{F}\right)^{2}\left(\frac{C_{F} C_{K}}{C_{F}+C_{K}}\right)^{2}} .
\end{aligned}
$$

Это выражение сравним с выражением для входного сопротивления $Z_{e}$ трансформированной схемы, показанной на рис. $8, b$ :

$$
Z_{e}=R_{e}(f)+\frac{1}{j \Omega C_{e}(f)}=R_{e}(f)-j \frac{1}{\Omega C_{e}(f)} .
$$

Здесь $R_{e}(f)$ и $C_{e}(f)$ - частотно зависимые параметры трансформированной схемы.

Приравнивая действительные и мнимые части выражений (П2) и (П3), получим

$$
\begin{gathered}
R_{e}(f)=R_{F} \frac{\left(\frac{C_{F}}{C_{F}+C_{K}}\right)^{2}}{1+\left(\Omega R_{F}\right)^{2}\left(\frac{C_{F} C_{K}}{C_{F}+C_{K}}\right)^{2}}, \\
\frac{1}{C_{e}(f)}=\frac{\frac{1}{\left(C_{F}+C_{K}\right)}+\Omega^{2} C_{K} R_{F}^{2}\left(\frac{C_{F}}{C_{F}+C_{K}}\right)^{2}}{1+\left(\Omega R_{F}\right)^{2}\left(\frac{C_{F} C_{K}}{C_{F}+C_{K}}\right)^{2}} .
\end{gathered}
$$

Учитывая правила перехода от механической модели к электрическому аналогу, в соответствии с которыми $r_{e}(f)=R_{e}(f)$ и $k_{e}(f)=\frac{1}{C_{e}(f)}$ (рис. 6), а также $r_{F}=R_{F}$, $k_{F}=\frac{1}{C_{F}}$ и $k_{K}=\frac{1}{C_{K}}$ (рис. 7), из (П4) и (П5) после преобразований получим искомые соотношения для параметров трансформированной модели, соответствующие выражениям (22) и (23):

$r_{e}(f)=r_{F} \frac{\left(\frac{k_{K}}{k_{F}+k_{K}}\right)^{2}}{1+\left(\frac{\Omega r_{F}}{k_{F}+k_{K}}\right)^{2}}, k_{e}(f)=\frac{k_{F} k_{K}}{k_{F}+k_{K}} \frac{1+\frac{\left(\Omega r_{F}\right)^{2}}{\left(k_{F}+k_{K}\right) k_{F}}}{1+\left(\frac{\Omega r_{F}}{k_{F}+k_{K}}\right)^{2}}$.

При этом модель Фойгта с параметрами трансформированной модели $r_{e}(f)$ и $k_{e}(f)$ будет полностью соответствовать модели Кельвина-Фойгта с параметрами $r_{F}, k_{F}$ и $k_{K}$.

\section{Конфликт интересов}

Авторы заявляют, что у них нет конфликта интересов.

\section{Список литературы}

[1] Физический энциклопедический словарь. Гл. ред. А.М. Прохоров. (Советская энциклопедия, М., 1983), $928 \mathrm{c}$.

[2] ISO 4664-2:2006. Rubber, vulcanized or thermoplastic: Determination of dynamic properties. Part 2: Torsion pendulum methods at low frequencies. (British Standards Institution, London, 2006), $12 \mathrm{p}$.

[3] А.И. Голованов, В.И. Митряйкин, В.А. Шувалов. Изв. вузов. Авиационная техника, 1, 66 (2009).

[4] Д.Р. Бленд. Теория линейной вязкоупругости (Мир, М., 1965), $199 \mathrm{c.}$

[5] Р. Кристенсен. Введение в теорию вязкоупругости (Мир, М., 1974), 340 c.

[6] Р.А. Ржаницын. Теория ползучести (Госстройиздат, М., 1968), $416 \mathrm{c}$.

[7] Н.Н. Малинин. Прикладная теория пластичности и ползучести. 2-ое изд. (Машиностроение, М., 1975), 400 с.

[8] Г.С. Варданян. Сопротивление материалов с основами теории упругости и пластичности (НИЦ ИН ФРА, М., 2015), $512 \mathrm{c}$.

[9] I.I. Argatov. Mathematical modeling of linear viscoelastic impact: Application to drop impact testing of articular cartilage. arXiv:1206.2681v1 [math.CA].12 Jun 2012.

[10] Н.А. Катанаха, А.С. Семенов, Л.Б. Гецов. Проблемы прочности 4, 143 (2013).

[11] B. Liu, L. Zhao, A.J.M. Ferreira. et al. Compos. Part B-Eng., 110 (1), 185 (2017)

[12] В.Н. Паймушин, В.А. Фирсов, Р.К. Газизуллин, С.А. Холмогоров, В.М. Шишкин. Механика композитных материалов, 55 (4), 635 (2019).

[13] В.Н. Паймушин, В.А. Фирсов, В.М. Шишкин. ПМТФ, 59 (3), 155 (2018).

[14] H. Sonnerlind. Теория и механизмы демпфирования в механике конструкций. [Электронный ресурс]: URL: https://www.comsol.ru/blogs/damping-in-structural-dynamicstheory-and-sources (дата обращения 16.12.2019).

[15] И.И. Вульфсон. Теория механизмов и машин, 3 (1), 44 (2005).

[16] Е. Скучик. Простые и сложные колебательные системы, пер. с англ. (Мир, М., 1971), 557 с.

[17] Б.М. Яворский, А.А. Детлаф, А.К. Лебедев. Справочник по физике для инженеров и студентов вузов. 8-ое изд. (ОНИКС, Мир и образование, М., 2008), 1056 с.

[18] Г. Ольсон. Динамические аналогии, пер. с англ. (Гос. издво иностр. лит-ры, 1947), 224 с.

[19] М.А. Сапожков. Электроакустика (Связь, М., 1978), $272 \mathrm{c}$.

[20] Ю.В. Максимов, Ю.С. Легович, Д.Ю. Максимов. Материалы 13 Межсдунар. конф. „Управление развитием крупномасштабных систем (MLSD”2020)“ (ИПУ РАН, M., 2020).

[21] М.В. Адов. Молодой ученый, 24.1 (104.1), 1 (2015). 